Eisenkölbl Closs AR, Franco Salinas BHM, Wessely Bogado LS.

Percepción de dos Comunidades Rurales y una Comunidad

ARTÍCULO ORIGINAL

Indígena sobre la Pandemia del COVID-19. Rev. Soc. cient. Parag.

ORIGINAL ARTICLE

2021;26(2):127-149.

https://doi.org/10.32480/rscp.2021.26.2.127

Recibido: 16/03/2021. Aceptado: 1/09/2021.

\title{
Percepción de dos Comunidades Rurales y una Comunidad Indígena sobre la Pandemia del COVID-19
}

\section{Perception of Two Rural Communities and one Indigenous Community on the COVID-19 Pandemic}

\author{
Alicia Raquel Eisenkölbl Closs ${ }^{1 *}$ (D), Bianca Herenia Margarita Franco \\ Salinas $^{1}$ (D) Lauria Soledad Wessely Bogado ${ }^{1}$ \\ ${ }^{1}$ Asociación Pro Cordillera San Rafael (PRO COSARA). Hohenau, Itapúa, Paraguay.
}

Autor correspondiente: aeisenkolbl1@gmail.com

Resumen: Ante la presencia del COVID-19, se identificó que en Paraguay la información de generación propia es escasa y en ocasiones, falsa; aun así, es diseminada por los medios de comunicación, a ello se suma que, en poblaciones rurales, la información sobre como el coronavirus afecta la salud es insuficiente y los comunicadores de estas zonas no están capacitados en la difusión de este tipo de noticias. La investigación se desarrolló en dos comunidades rurales y una comunidad indígena del distrito de Alto Vera, Itapúa, Paraguay. El objetivo principal fue elaborar un diagnóstico participativo para determinar la percepción de las comunidades de Poncho, Caraguata y Mberu Pirapo'i sobre la pandemia del COVID-19. Se aplicó una investigación con enfoque mixto, en lo cuantitativo se trabajó con un diseño no experimental, transaccional descriptivo, en lo cualitativo con grupos focales (personal de salud, líderes/referentes comunitarios y autoridades). Al concluir la investigación, se comprobó que la población accede a informaciones sobre el COVID-19 principalmente a través de medios radiales, seguido de la televisión y las redes sociales; sin embargo, no pueden confirmar si la información recibida a través de esta última es real o no. Además, se identificó una disminución en la implementación de medidas sanitarias en el área; la información obtenida es insumo necesario para establecer una estrategia comunicativa utilizando tecnologías existentes en la comunidad, a fin de permitir una comunicación fluida y real sobre la pandemia del COVID-19 a ser implementada en el área de estudio y con posibilidad de ser replicada.

Palabras clave: COVID-19, población rural, percepción, comunicación. 
Abstract: In view of the presence of COVID-19, it was identified that in Paraguay, selfgenerated information is scarce and sometimes false; even so, it is disseminated by the media, in addition to the fact that, in rural populations, information on how the coronavirus affects health is insufficient and communicators in these areas are not trained in the dissemination of this type of news. The research was carried out in two rural communities and one indigenous community in the district of Alto Vera, Itapúa, Paraguay. The main objective was to elaborate a participatory diagnosis to determine the perception of the communities of Poncho, Caraguata and Mberu Pirapo'i on the COVID-19 pandemic. A mixed approach research was applied: a non-experimental, transactionaldescriptive design was used for the quantitative part, and a focus group (health personnel, community leaders/referents and authorities) for the qualitative part. At the conclusion of the research, it was found that the population accesses information on COVID-19 mainly through radio media, followed by television and social networks; however, they cannot confirm whether the information received through the latter is real or not. In addition, a decrease in the implementation of sanitary measures in the area was identified; the information obtained is necessary input to establish a communication strategy using existing technologies in the community, in order to allow a fluid and real communication about the COVID-19 pandemic to be implemented in the study area and with the possibility of being replicated.

Keywords: COVID-19, rural population, perception, communication.

\section{INTRODUCCIÓN}

El 2020 fue un año que nos encontró sin una preparación previa para sobrellevar una pandemia, esto quedó demostrado por los estragos que causó el nuevo coronavirus. Esta "es una extensa familia de virus que pueden causar enfermedades tanto en animales como en humanos. En estos últimos, se sabe que causan infecciones respiratorias que pueden ir desde el resfriado común hasta enfermedades más graves como el síndrome respiratorio de Oriente Medio (MERS) y el síndrome respiratorio agudo severo (SRAS). El coronavirus que se ha descubierto más recientemente causa la enfermedad por coronavirus denominada COVID-19"(1).

Las primeras informaciones que se recibían de esta enfermedad fueron notificadas en Wuhan (China) el 31 de diciembre de 2019(2). Desde el inicio de la pandemia, la OMS se encuentra colaborando estrechamente con expertos mundiales, gobiernos y asociados para ampliar rápidamente los conocimientos científicos sobre este nuevo virus, rastrear su propagación y 
virulencia, y asesorar a los países y personas sobre las medidas para proteger la salud y prevenir la propagación de esta enfermedad(3).

Ante la pandemia, Paraguay actuó rápidamente, por lo que se pudo evitar la propagación masiva del virus. Las medidas exitosas que llevaron a reducir los contagios fueron a) aislamiento preventivo general a nivel nacional, toque de queda nocturno, b) aislamiento geográfico, esto muy favorecido por la baja densidad de la población y c) complementado por el oportuno cierre de fronteras terrestres ${ }^{(4)}$; y por último podemos mencionar la implementación de la cuarentena inteligente, sin olvidar que se tuvo el logro a pesar del sistema de salud pública debilitado con que cuenta el Paraguay.

Se pudo evidenciar que el rol de los medios de comunicación ante la difusión de la información del desarrollo de la pandemia no era adecuada, identificando específicamente estas situaciones a) "gran parte de la colectividad paraguaya ha caído en la diseminación de información falsa incluso ya desde antes del ingreso del virus al país, lo que ha generado una rápida reacción de neurosis en la sociedad, decantando en acciones relacionadas con compras compulsivas, xenofobia, prácticas riesgosas de cuidado sanitario", así también, se determinó b) que los principales medios masivos de comunicación contaban con una muy escasa -por no decir nulaproducción propia de noticias basadas en fuentes científicas primarias, denotando una redacción basada en la traducción directa de otros periódicos extranjeros ${ }^{(5)}$.

Por todos estos aspectos, se considera que es importante contar con un instrumento de establecimiento e implementación de una estrategia de comunicación a partir de la utilización de tecnologías existentes en la comunidad para sensibilizar a la población rural e indígena sobre la prevención y protección de la salud ante el COVID-19.

\section{METODOLOGÍA}

\subsection{Localización}

El área de estudio se compone por las localidades de Poncho, Caraguata, la comunidad indígena de Mberu Pirapo'i y área de influencia a estas comunidades (Figura 1), ubicadas en el distrito de Alto Verá, departamento de Itapúa. 


\subsection{Población y muestra}

La población de estudio, estuvo compuesta por 220 familias, de estas 120 corresponden a familias de Poncho, 70 de Caraguata y 30 a la comunidad indígena. La muestra fue de 103 familias, considerando a la comunidad indígena una familia.

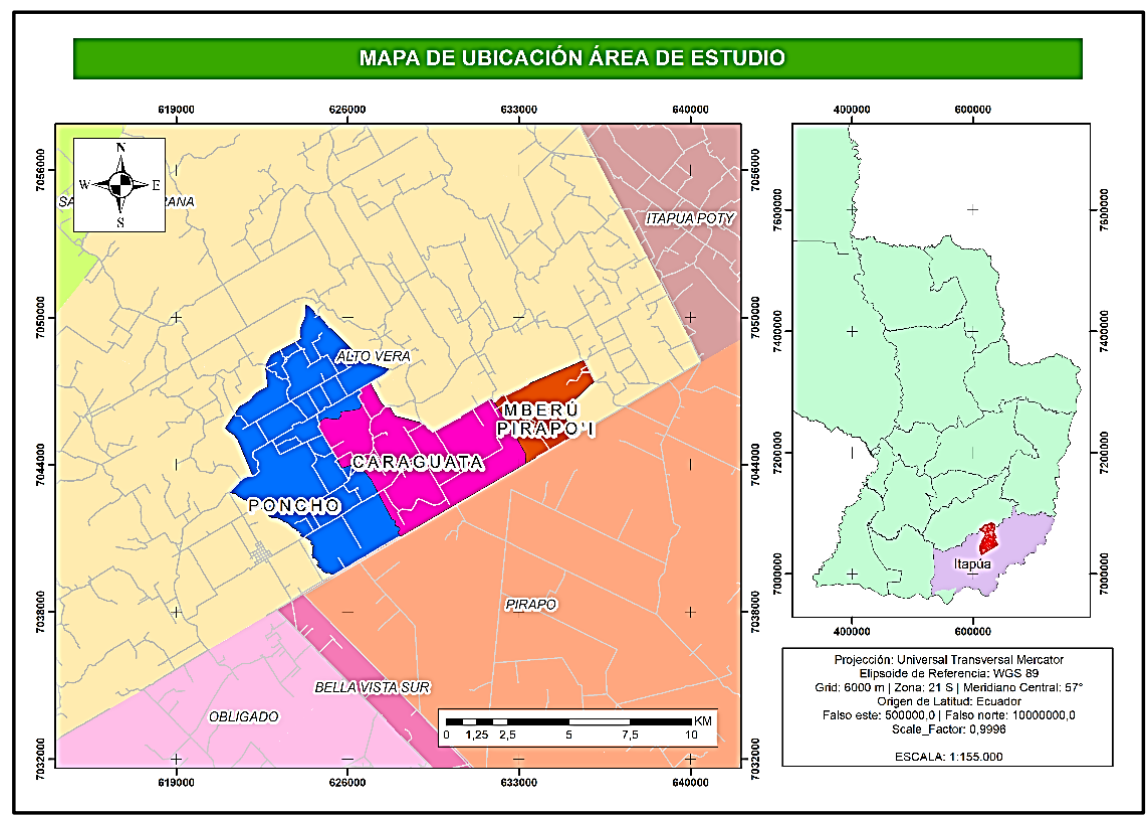

Figura 1. Mapa de ubicación del área de estudio.

\subsection{Diseño de la investigación}

La investigación tuvo un enfoque mixto: cuali-cuantitativo. Los enfoques mixtos representan un conjunto de procesos sistemáticos, empíricos y críticos de investigación e implican la recolección y el análisis de datos cuantitativos y cualitativos, así como su integración y discusión conjunta, para realizar inferencias producto de toda la información recabada y lograr un mayor entendimiento del fenómeno bajo estudio. En lo cuantitativo se trabajó con un diseño no experimental, transaccional descriptivo en esta primera fase se recolectaron y analizaron datos para explorar un fenómeno, donde se generó una base de datos. En lo cualitativo se trabajó con grupos focales, con el fin de recolectar y analizar información de manera a poder responder interrogantes de la investigación(6). 
Rev. Soc. cient. Parag. 2021;26(2):127-149

\subsection{Recolección y procesamiento de la de la información}

Con la intención de reproducir la estructura del colectivo se definió la recolección de información de la siguiente manera: a) Se elaboró un formulario de encuesta y cuestionario de entrevista (6), las mismas fueron previamente aprobadas por las autoridades municipales y de salud del distrito. b) Se determinó el muestreo en 103 familias, y se distribuyeron las encuestas considerando la población existente en cada localidad, es así que se realizaron 62 encuestas en Poncho, 40 Caraguata y 1 encuesta individual al cacique de la comunidad indígena Mberú Pirapo'i. Es importante destacar que, en la comunidad indígena, por cuestiones culturales se aplicó la encuesta únicamente al cacique, líder local, integrando los datos. Así también, se le realizó otra encuesta en donde respondió de manera general los datos de las familias que residen en su comunidad. c) Aplicación de la encuesta. d) Las entrevistas fueron realizadas de forma intencionada y fueron dirigidas a referentes comunitarios y de salud.

Para el procesamiento de la información se utilizó la metodología sugerida por ${ }^{(6)}$ a) Primera fase: preparación. Es el momento previo a la toma de información, en el cual se planifican los aspectos organizativos de la misma, como son los objetivos, redacción de preguntas guía y convocatoria, b) Segunda fase: apertura. Es la fase cuando se está con el entrevistado en el lugar de la cita, en el que se plantean los objetivos que se pretenden con la entrevista, el tiempo de duración. También, es el momento oportuno para solicitar el consentimiento de grabar o filmar la conversación, c) Tercera fase: desarrollo. Constituye el núcleo de la toma de información, en el que se intercambia información siguiendo la guía de preguntas con flexibilidad. Es cuando el entrevistador hace uso de sus recursos para obtener la información que se requiere, d) Cuarta fase: cierre. Es el momento en el que conviene anticipar el final la toma de información para que el entrevistado recapitule mentalmente lo que ha dicho y provocar en él la oportunidad de que profundice o exprese ideas que no ha mencionado. Se hace una síntesis de la conversación para puntualizar la información obtenida y finalmente se agradece al entrevistado su participación en el estudio.

La recolección de la información fue de forma aleatoria en el caso de las encuestas e intencionada para las entrevistas, los sujetos de estudio (población afectada al estudio) fueron escogidos de acuerdo a ciertos criterios de selección acordes con los objetivos de la investigación. Según(7) "la entrevista es una estrategia para hacer que la gente hable sobre lo que sabe, piensa y cree, esta información suele referirse a la biografía, al sentido de los hechos, a sentimientos, opiniones y emociones, a las normas o estándares de acción, y a los valores o conductas ideales que van surgiendo 
de la interacción entre el entrevistado y el entrevistador. Se utilizaron encuestas y entrevistas semiestructuradas ${ }^{(6)}$ con preguntas formuladas en función a los objetivos del estudio, las mismas fueron aplicadas a la población, líderes y autoridades. Cada formulario se compuso por 7 bloques de contenido y 28 ítems.

Posteriormente, se analizaron los datos cualitativos y cuantitativos, los cuales permitieron extraer interpretaciones relacionadas a los objetivos de esta investigación, se obtuvieron a partir de los resultados de bloques de encuestas y entrevistas aplicadas a los habitantes provenientes de las zonas de estudio.

Los datos correspondientes a hacinamiento, se establecen por medio de la relación entre la cantidad de personas que residen en la vivienda y la cantidad de habitaciones destinadas a dormitorios en la misma(8) así también, mencionan que el índice para determinar si una vivienda se encuentra o no en situación de hacinamiento es de 1.5 a 2 .

Para el procesamiento de los datos y elaboración de los gráficos correspondientes, se utilizaron planillas electrónicas. Luego, los resultados fueron confrontados con la teoría de base de la investigación para su correspondiente análisis y contrastación con bibliografía, con lo cual posteriormente se elaboró un resultado integral, es decir, globalizado de la investigación

\section{RESULTADOS Y DISCUSIÓN}

Con el fin principal de analizar el comportamiento de estas comunidades rurales, se ha aplicado un total de 103 encuestas a pobladores del área. En la figura 2 , se puede observar la distribución de la aplicación de las mismas en cada comunidad seleccionada.

\subsection{Situación Económica y Vivienda}

En el área de estudio se pudo verificar que los ingresos de la mayor parte las familias (46\%) son menores a Gs. 500.000 mensuales, seguido por un $19 \%$ que percibe menos de Gs. 1.000.000. La situación económica en el área de estudio no difiere mucho de otras áreas rurales, la $\mathrm{FAO}{ }^{\left({ }^{9}\right)}$ hace mención que en la Encuesta Permanente de Hogares (2019) se identificó que el 23,5\% de la población paraguaya se encontraba en situación de pobreza. Esto significa que alrededor de 1,657 millones de personas residen en hogares cuyos ingresos son inferiores al costo de una canasta básica de consumo estimado para dicho año. Históricamente, la pobreza ha sido mayor en el área rural, si 
bien hay pobreza rural en todos los departamentos del país, el 62,7\% se concentra en cinco de ellos: Caaguazú, San Pedro, Itapúa, Caazapá y Concepción. La pobreza extrema rural también se concentra en estos departamentos, a los que hay que añadir Guairá y Canindeyú.

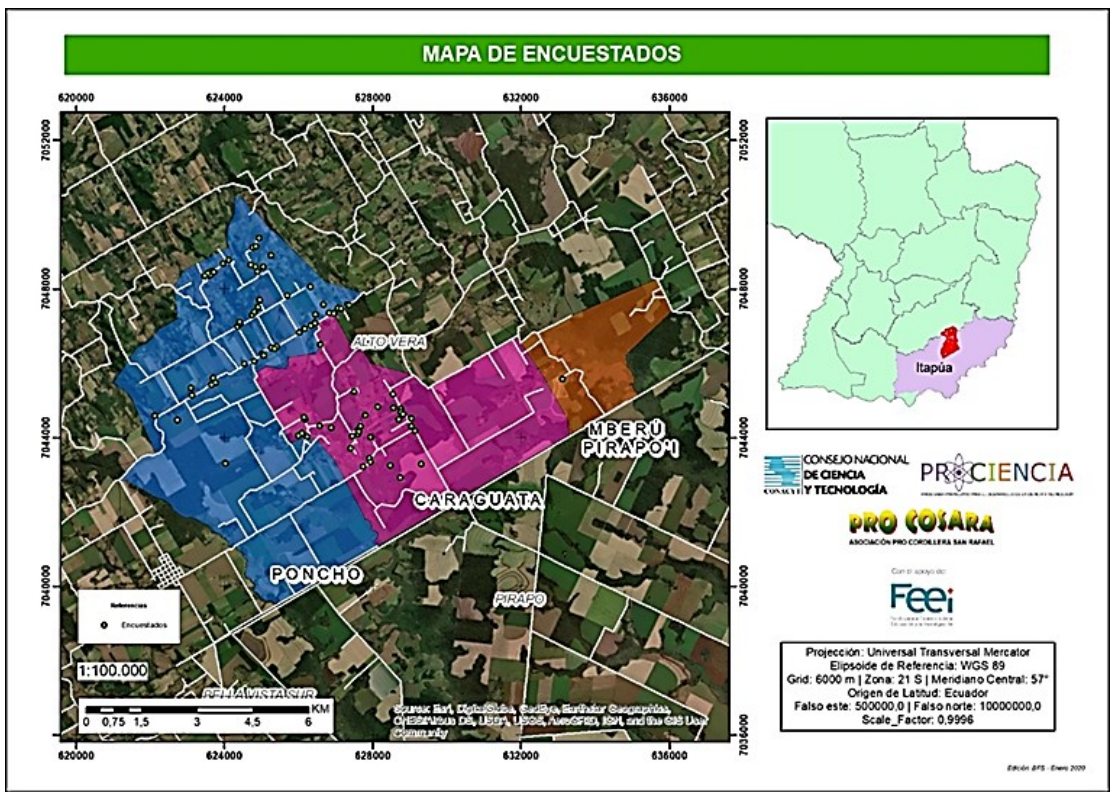

Figura 2. Mapa de Encuestados en Poncho, Caraguata y Mberu Pirapo'i, 2021.

Con respecto a la situación de vivienda, en la comunidad de Poncho, se han encuestado a un total de 62 familias, que representan a 218 personas; con los resultados obtenidos se puede mencionar que la mayor parte de las viviendas cuenta con 2 habitaciones, y con un promedio de 4 residentes. Así también, en Caraguatá fueron aplicadas 40 encuestas, totalizando así a 183 personas; en esta comunidad hay un promedio de 5 personas por casa y 2 habitaciones.

Esto a simple vista no representa ningún problema de hacinamiento, ya que Spicker y Álvarez ${ }^{(8)}$ mencionan que el índice para determinar si una vivienda se encuentra o no en situación de hacinamiento es de 1.5 a 2, este se establece por medio de la relación entre la cantidad de personas que residen en la vivienda y la cantidad de habitaciones destinadas a dormitorios en la misma(10). Sin embargo, haciendo un análisis más exhaustivo, se pudo identificar que sí existen familias con un umbral de 
hacinamiento en las comunidades. Pues según un informe de la Comisión Económica para América Latina y el Caribe y el Fondo de las Naciones Unidas para la Infancia(11), se considera hacinamiento moderado cuando existen de 3 a 4 personas por dormitorio y hacinamiento severo de 5 o más personas.

Como se observa en la Figura 3, en la comunidad de Poncho se han identificado 17 familias con hacinamiento moderado, mientras que en Caraguata 5 de las viviendas encuestadas presentan un hacinamiento moderado y 1 de ellas hacinamiento severo. De igual modo, en la comunidad de Mberu Pirapo'i se identificó que existe hacinamiento severo, lo que representa un mayor riesgo de contagio y no tan buenas condiciones de vida.

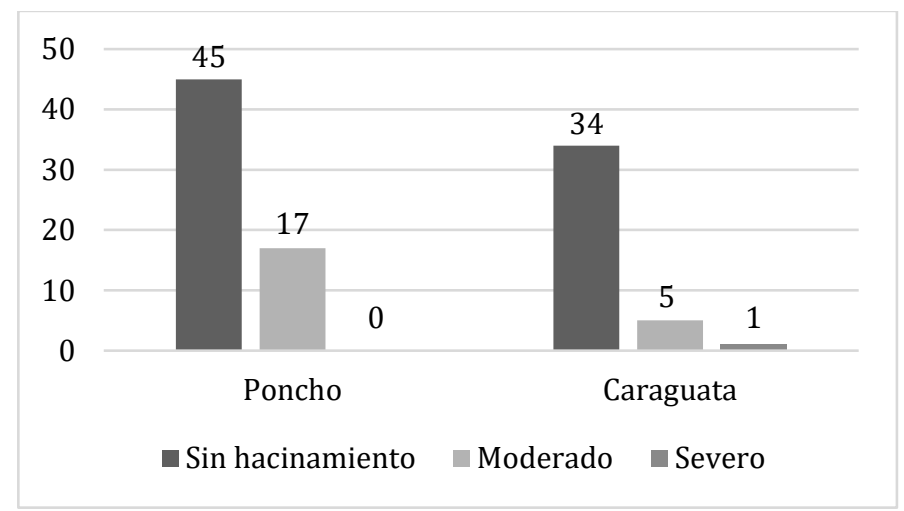

Figura 3. Umbral de hacinamiento comunidades Poncho y Caraguata, 2021.

Según el Plan de Desarrollo 2030(12), el déficit de vivienda afecta a una importante franja de la población (desde los grupos más vulnerables hasta la clase media), siendo la oferta de vivienda social insuficiente, pues se tiene un déficit actual de 1.100.000 viviendas. Identificar las condiciones de vivienda y hacinamiento, es fundamental para realizar proyecciones de los contagios; de igual modo, determinar estos niveles en las comunidades rurales es esencial pues con ello se puede identificar si el contagio podrá ser controlado, así como también, permite conocer si, en el caso de presentarse un caso positivo dentro de la familia, se podrá contar con las medidas correctas para realizar el aislamiento de la o las personas infectadas con el virus. 
Rev. Soc. cient. Parag. 2021;26(2):127-149

\subsection{Acceso a Servicios Básicos y Medidas de Prevención}

El acceso y gestión segura de servicios como agua y saneamiento básico, son esenciales para hacer frente a la pandemia del COVID-19. De igual modo, prácticas adecuadas de higiene y de gestión de residuos permiten prevenir la propagación de innumerables enfermedades infecciosas ${ }^{(13)}$ es muy importante resaltar que todos los encuestados tienen acceso a algún tipo de fuente de agua; sin embargo, la provisión de la misma varía.

En Poncho, la población se provee de agua a través de pozos con bomba, Junta de Saneamiento y pozos sin bomba. Mientras que, en Caraguata, una gran mayoría se provee a través de la Junta de Saneamiento y en menor proporción de la red comunitaria; en la comunidad indígena de Mberu Pirapo'i, la provisión de agua se da a través también de la red comunitaria de agua (Figura 4).

En relación al saneamiento básico (Figura 5), en la comunidad Poncho el mayor número cuenta con letrina sin techo/puerta, de igual modo se han identificado en semejante número, familias que cuentan con cámara séptica/pozo ciego y letrina de hoyo. Mientras que, en Caraguata del total de encuestados, 19 familias utilizan cámara séptica/pozo ciego, seguido de letrina de hoyo, letrina sin techo/puerta; en Mberu Pirapo'i, las familias poseen letrina de hoyo. Esta situación se plantea en el Plan de Desarrollo 2030, donde el déficit de agua y saneamiento afecta principalmente a las zonas marginales periurbanas y en el área rural a las poblaciones en situación de pobreza, las comunidades dispersas, los asentamientos y las poblaciones indígenas.

Teniendo en cuenta el ODS 6(14) "Garantizar la disponibilidad de agua y su gestión sostenible y el saneamiento para todos", queda plenamente evidenciado que se tienen complicaciones con el logro de este objetivo, en especial en los siguientes puntos: 6.1 Lograr el acceso universal y equitativo al agua potable a un precio asequible para todos; 6.2 Lograr el acceso a servicios de saneamiento e higiene adecuados y equitativos para todos y poner fin a la defecación al aire libre, prestando especial atención a las necesidades de las mujeres y las niñas y las personas en situaciones de vulnerabilidad(15). 


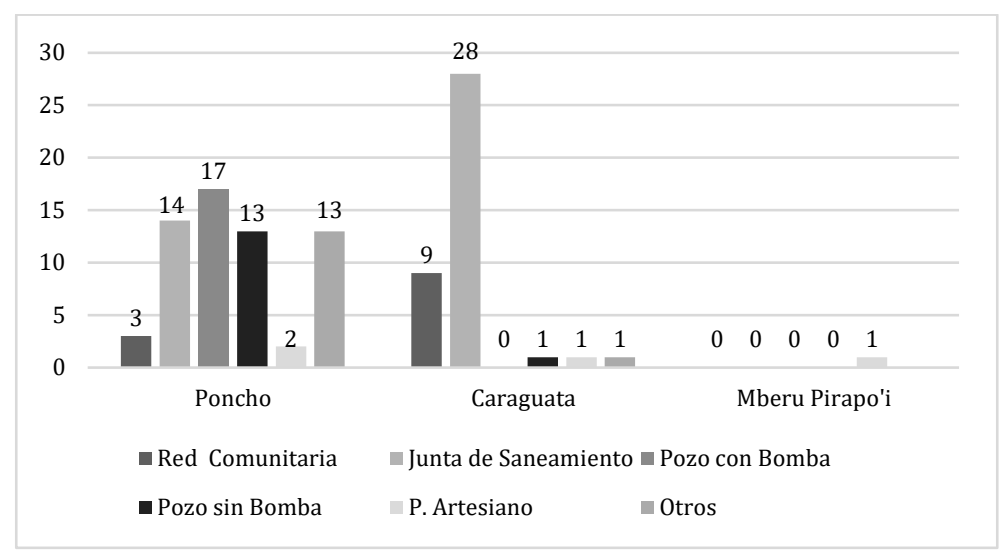

Figura 4. Provisión de Agua en las Viviendas de los Encuestados de las 3 comunidades, 2021.

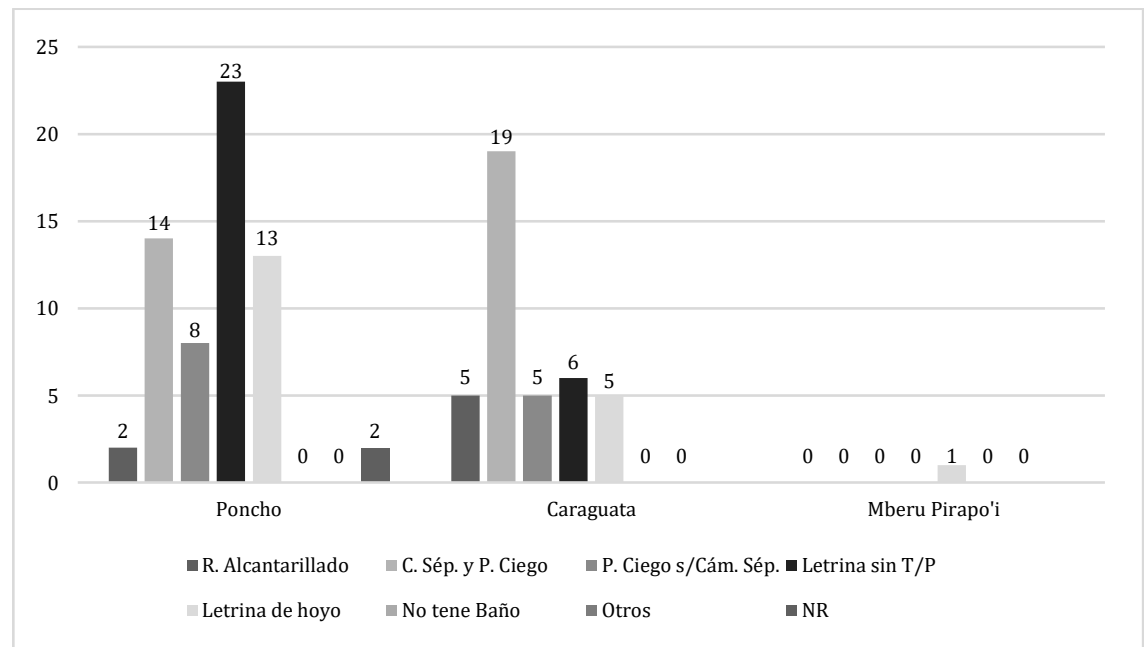

Figura 5. Saneamiento Básico en las 3 comunidades, 2021.

De igual modo, en relación a la disposición final de residuos sólidos, se resalta que el municipio no cuenta con el servicio de recolección ni tampoco con un relleno sanitario. Sobre este punto, se vio que la gran mayoría de los encuestados utilizan la incineración como método de disposición final, seguido por un porcentaje mucho menor que entierra los residuos en sus 
propiedades (Figura 6), ambas prácticas son consideradas no sostenibles y a su vez una debilidad importante de gran parte de los municipios del Paraguay ${ }^{(12)}$.

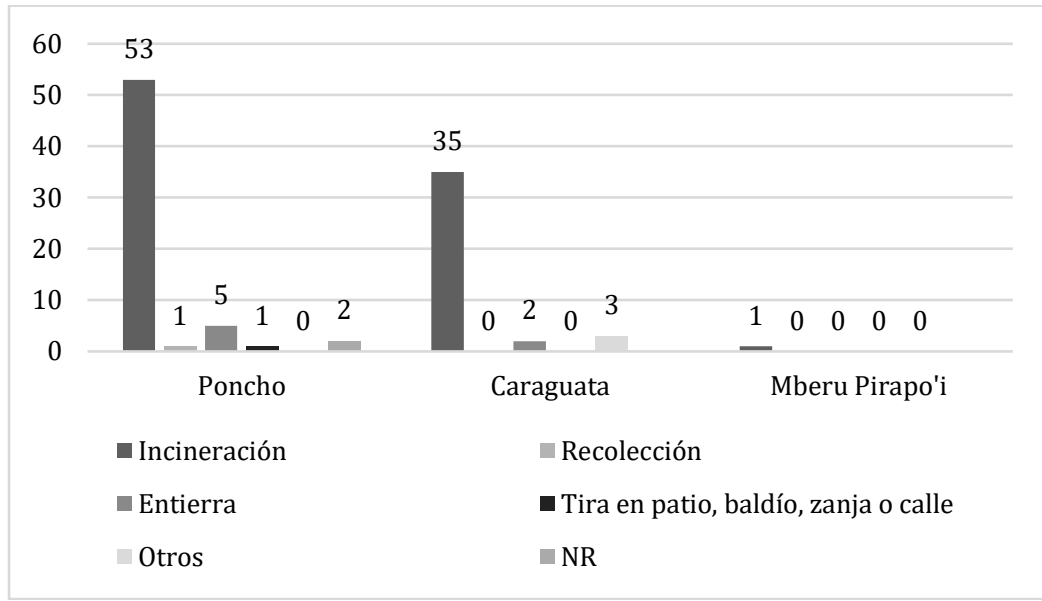

Figura 6. Disposición Final de Residuos en las 3 comunidades, 2021

Nuevamente queda expuesto que Paraguay sigue teniendo dificultades para cumplir la Estrategia 1.4 - Hábitat Adecuado y Sostenible Mejoramiento, del acceso y las condiciones de la vivienda y el hábitat, los servicios básicos, saneamiento, provisión de agua, calidad de aire, suelo y tratamiento de residuos(12), lo más preocupante de esta situación es que las comunidades se encuentran cercanas al centro urbano de referencia.

\subsection{Acceso a Servicios de salud}

En relación a los factores de riesgo, el Ministerio de Salud Pública y Bienestar Social(16), menciona que personas mayores de 60 años y con condiciones de salud subyacentes son más propensas a sufrir complicaciones graves por el COVID-19, esta es la población de riesgo que debe presentar especiales cuidados. En el área de estudio se pudo identificar que la población de esta franja etaria no supera el $30 \%$.

Se han identificado a 25 personas Figura 7 que padecen enfermedades de base, entre ellas se resaltan que la patología que afecta a un mayor número de personas es la diabetes, mientras que también se han identificado personas con padecimientos como presión alta, colesterol, hipertensión, asma. Es importante resaltar que, en la comunidad indígena no se han identificado personas con alguna enfermedad de base. 
Rev. Soc. cient. Parag. 2021;26(2):127-149

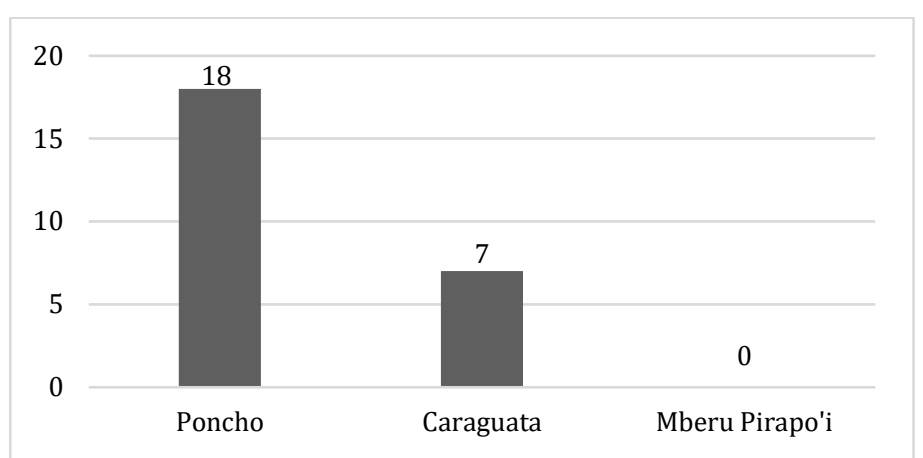

Figura 7. Número de Personas con Enfermedades de Base en las 3 comunidades, 2021.

Es importante destacar que, estudios han concluido que las patologías de base (sin considerar la edad) como hipertensión arterial, diabetes, problemas cardiacos o antecedentes de enfermedades respiratorias, hacen que las personas con estas condiciones sean mucho más susceptibles a la infección y son más propensos a desarrollar cuadros de mayor gravedad.

En cuanto a la accesibilidad que tienen los pobladores a los servicios de salud (Figura 8), se pudo verificar que un alto porcentaje de la población encuestada tanto de la localidad de Poncho (89\%) y en la localidad de Caraguata (63\%), concuerdan que tienen mejor acceso de servicios en el Puesto de Salud, siendo el mismo el más cercano.

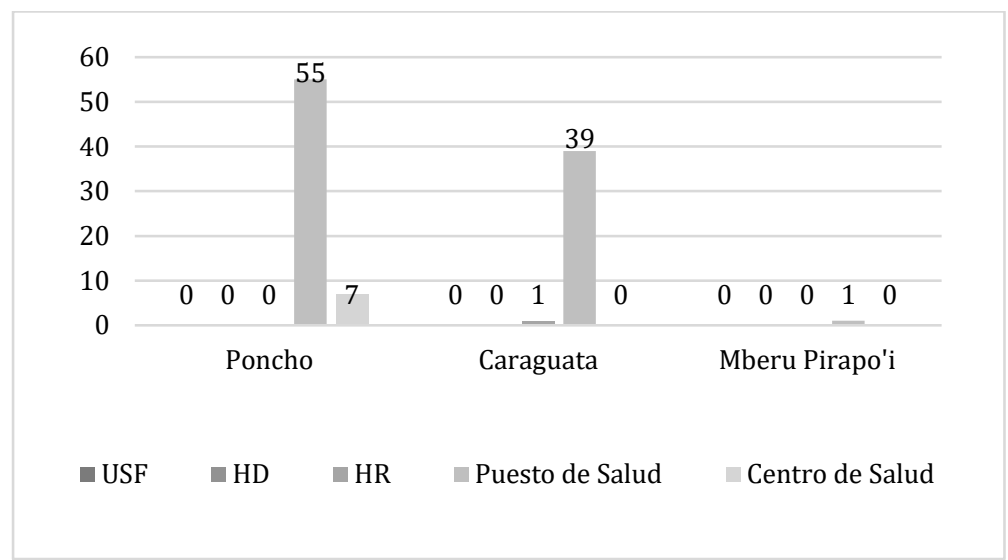

Figura 8. Acceso a Servicios de Salud en las 3 comunidades, 2021. 
De igual modo, se consultó sobre la regularidad con la que se asiste a los servicios de salud (Figura 9), para ello se consideró regular 1 vez al mes; en los resultados se observan que, en Poncho una gran mayoría de los encuestados (82\%) asiste regularmente a algún servicio de salud, mientras que 7 de los encuestados manifestaron que no asisten regularmente a algún servicio de salud.

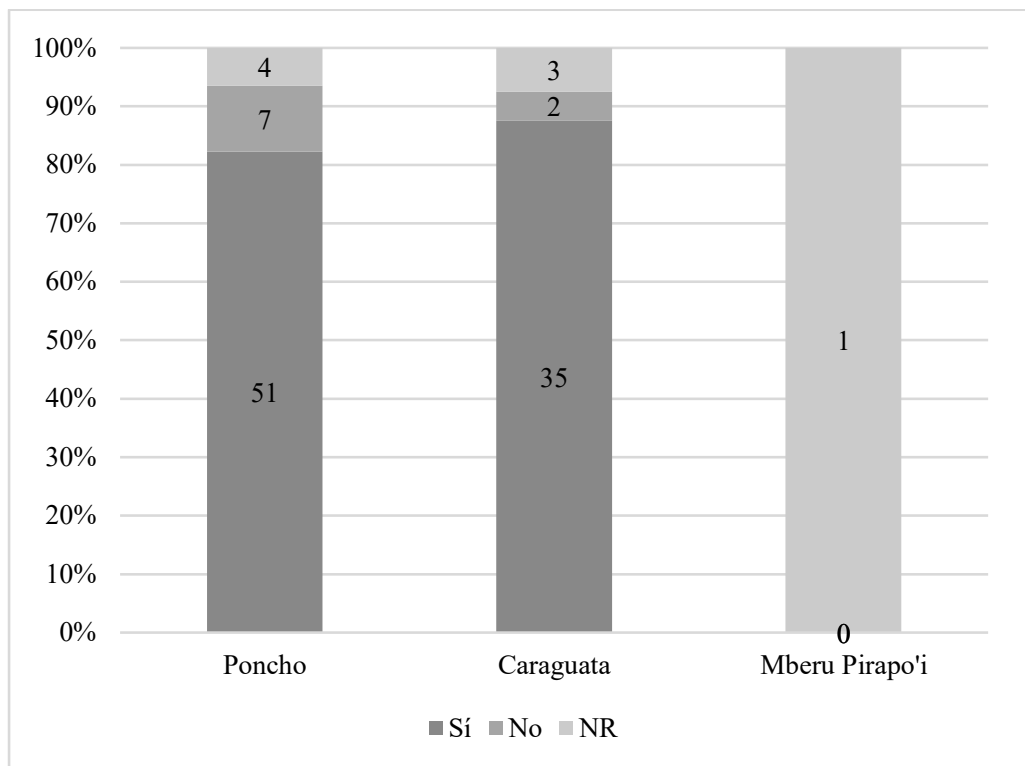

Figura 9. Frecuencia de Asistencia a Servicios de Salud en las 3 comunidades, 2021.

Entre las acciones más eficientes para evitar la propagación de enfermedades, como el COVID-19 se puede destacar al lavado de manos y la desinfección de áreas comunes(17). El 19 de marzo el Ministerio de Salud toma el mando operativo de todo el sistema de salud pública, con facultad de toma de decisiones y ejecución de acciones, operativas y estratégicas de alcance nacional, en coordinación con todas las instituciones sanitarias del país(18).

Se pudo identificar que, en el área de estudio, se logró instalar la aplicación de los protocolos sanitarios indicados por el Ministerio de Salud y Bienestar Social (MSyBS) (higiene de manos, utilización de tapabocas, distanciamiento social), así también, se cumplió con la realización de la cuarentena y se implementaron controles sanitarios de ingreso de personas de otros lugares del país o del exterior, se tuvo apoyo municipal con carteles informativos. 
A su vez el personal del Puesto de Salud cumplió como ente difusor de la información, según lo establecido por la Resolución $N^{\circ} 90$ "Medidas para mitigar propagación del COVID-19", Resolución $N^{\circ} 91$ "Se aprueba plan nacional de respuesta a virus respiratorios 2020", Resolución $N^{\circ} 99$ "Aislamiento preventivo", Resolución № 112 "Registro obligatorio de personas provenientes del extranjero en plataforma digital"(19).

Se evidenció que el apoyo del Gobierno Nacional, se dio a través de la provisión de insumos de bioseguridad al Puesto de Salud a través del MSPyBS, así también hubo algunas personas beneficiadas con los subsidios estatales ${ }^{(18)}$ en tanto se tuvo apoyo Municipal mediante la provisión de víveres a comunidades cuyos ingresos se vieron afectados debido a que no podían movilizarse para realizar la venta de sus productos agropecuarios.

Con respecto a la utilización de agua y jabón, el 100\% de los encuestados en las 3 comunidades ha respondido que sí utiliza estos elementos para higienizarse.

Como se observa en la figura 10 gran número de familias cuentan con elementos de desinfección en la vivienda, como lavandina y alcohol, todos los encuestados en las 3 comunidades utilizan algún tipo de medida de bioseguridad establecida por el MSPyBS, la más utilizada es la higiene de manos, seguida por el uso de tapabocas y desinfección del hogar (Figura 11).

De igual modo, a fin de determinar si estas informaciones son útiles, se procedió a consultar si en su rutina diaria incluyen hábitos de higienización, desinfección y protección contra el virus; sobre este punto, el $100 \%$ de los encuestados en todas las comunidades han respondido que sí.

\subsection{Percepción de Líderes y Referentes Comunitarios sobre el COVID-19}

A la consulta de cómo, desde su papel en la comunidad, están aportando a la situación la mayoría de los entrevistados consideran que el principal aporte que dan es el cumplimiento de los protocolos sanitarios (uso de tapabocas, alcohol y el lavado de manos) así instituidos por el MSyBS el 04 de marzo realiza la socialización del "Plan de Respuesta Nacional-COVID-19"(18). 
Rev. Soc. cient. Parag. 2021;26(2):127-149

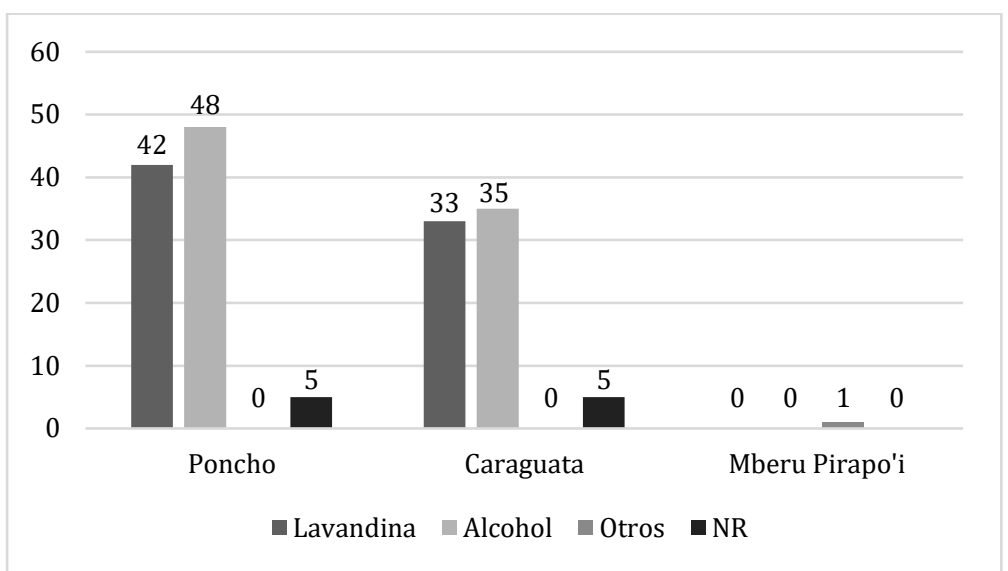

Figura 10. Elementos de Desinfección con los que se Cuentan en las Viviendas de los Encuestados, en las 3 comunidades, 2021.

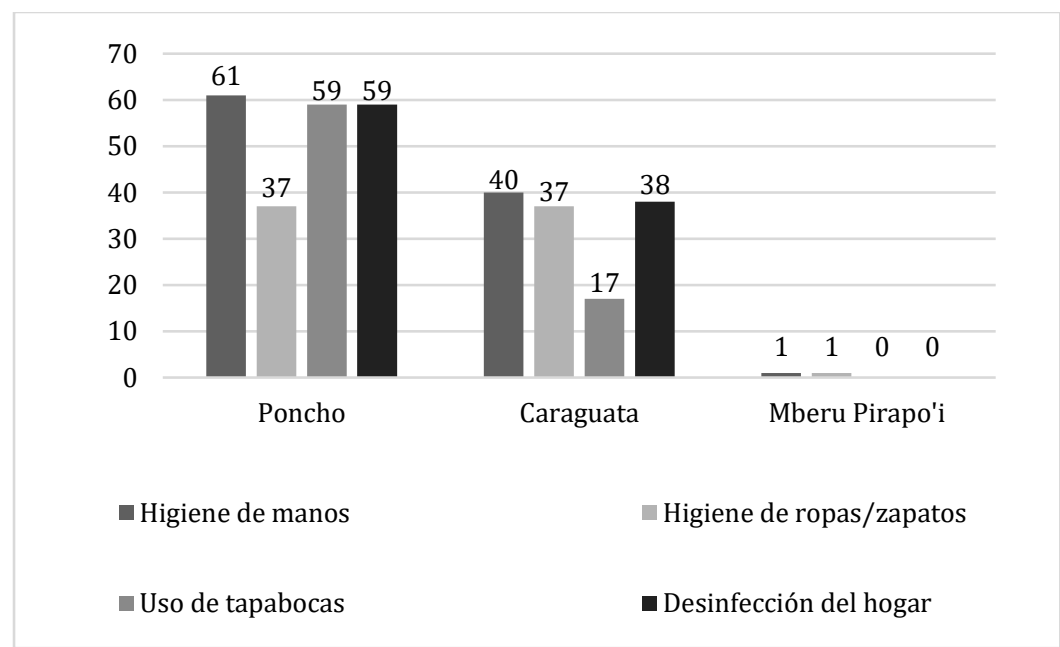

Figura11. Medidas de Bioseguridad que Utilizan las Familias Encuestadas en las 3 Comunidades, 2021.

A su vez, se consultó si conocían si el Estado proveía de algún tipo de ayuda para hacer frente al virus o si se autogestionan en los recursos; sobre este punto se mencionó que no se tiene apoyo local directo del Estado, solo se tienen algunas personas beneficiadas con los subsidios estatales(18). Sin embargo, sí se identificó el apoyo municipal a la comunidad, a través de la 
provisión de recursos al Puesto de Salud y distribución de víveres a las comunidades.

Así también, todas las respuestas coincidían en que al inicio de la pandemia y al imponerse las medidas de bioseguridad, la población cumplió correctamente los protocolos para evitar la propagación del virus, estas medidas sanitarias adoptadas fueron estrictas. Sin embargo, actualmente se observa un relajo en la aplicación de las mismas, se podría decir que es por la baja cantidad de casos positivos, es por ello que continuar con la concienciación constante es sumamente importante para evitar el contagio.

Se puede apreciar el uso de tapabocas por parte de algunos de los pobladores, pero la medida que gran número de la población ha dejado de implementar es el distanciamiento social; el lavado de manos y uso de alcohol solo se da en instituciones y locales donde lo exigen. Los entrevistados concuerdan en su totalidad que existe un relajo en la aplicación de medidas sanitarias y que es sumamente importante contar con capacitación constante para así evitar que el virus se propague con más fuerza en el área de estudio.

La situación de relajo puede deberse a que: a) el área registró escasos casos de personas infectadas con el coronavirus, y esto hizo que la personas dejen de cuidarse, b) la falta de información o falta de conciencia del poder de infestación del virus.

Con lo anteriormente expuesto, se puede considerar que la población ya no tiene la conciencia suficiente del peligro que acarrea la pandemia del COVID19 , se han dejado de cuidar y por ende se ha abierto nuevamente la brecha de contagio en el área de estudio.

Como es sabido, durante la ocurrencia y epidemias y otras crisis, las comunidades indígenas y en especial las mujeres y niñas, se ven más afectados por las consecuencias de estas situaciones. Así también, las vivir en comunidades aisladas, los servicios básicos y, en especial los de salud, son precarios y con capacidad limitada ${ }^{(20)}$.

Con respecto a la adaptación de los pueblos indígenas a la pandemia del COVID-19, estos deben poner en práctica el cumplimiento de los Protocolos del Ministerio de Salud y las medidas sanitarias impuestas por el Gobierno Nacional.

En la entrevista realizada a referentes y líderes comunitarios, únicamente aquellos que cumplían funciones dentro de la Municipalidad, manifiestan que se realizaron capacitaciones a las comunidades indígenas a por parte de 
la Gobernación de Itapúa a través de la Secretaría de Asuntos Indígenas y responsables del Puesto de Salud, esta actividad fue realizada con apoyo municipal.

Sin embargo, los referentes comunitarios entrevistados indican que no conocen la situación de las comunidades indígenas en relación a la pandemia, así también desconocen qué tipo de información disponen y si los mismos fueron capacitados con respecto al COVID-19.

Se resalta que los puntos más trabajados en las comunidades en relación al COVID-19 por parte del Puesto de Salud fueron los siguientes puntos: a) Búsqueda de relacionamiento interpersonal, visitas a las comunidades para concienciación de los mismos sobre el tema de la pandemia y su implicancia, b) Concienciación sobre evitar el traslado a otras comunidades. En el Puesto de Salud y USF, indican que cuentan con Orientación General en base a los protocolos y estrategias establecidos por el MSPyBS para trabajos en las comunidades indígenas, según lo expuesto en la Ley $N^{\circ}$ 5469/2015 De la Salud Indígena, artículo $4^{\circ}$. El Estado, a través del Ministerio de Salud Pública y Bienestar Social, asegurará los medios y recursos necesarios para que los Pueblos Indígenas participen efectivamente en el proceso de atención integral a su salud(21).

Sobre este punto, se puede mencionar que la Dirección Nacional de Salud de los Pueblos Indígenas (DINASAPI) y el Consejo Nacional de Salud de los Pueblos Indígenas (CONASAPI), en conjunto con el Ministerio de Salud y Bienestar Social, estableció una "Guía y Recomendaciones para la Prevención y Protección del COVID-19 Dirigidos a los Pueblos y Comunidades Indígenas del Paraguay"(22), en la misma se establecen los protocolos y directrices para que las comunidades indígenas adopten los mismos a fin de adaptarse a la "nueva forma de vivir".

Se identificó en la entrevista con el líder de la comunidad indígena estudiada escasa aplicación de protocolos sanitarios por parte de los indígenas, el uso de tapabocas prácticamente es la única medida de prevención que utilizan para prevenir el virus.

\subsection{Comunicación del COVID-19}

Como se puede observar en la figura 12 , el $41 \%$ del total de encuestados, utiliza con mayor frecuencia la radio, seguido TV al aire para acceder a las informaciones. Así también, se identificó un menor número que utiliza TV cable para poder acceder a informaciones, y de igual modo, el 8\% de los encuestados utiliza el acceso a internet (redes sociales). 
Sin embargo, un estudio realizado por el Ministerio de Tecnologías de la Información y Comunicación (23), con la finalidad de conocer la percepción de las familias paraguayas en cuanto a las informaciones sobre el Coronavirus, concluyó que para la mayor parte de las 1049 familias encuestadas en ese estudio, la TV es el principal medio de comunicación que utilizan para acceder a las informaciones y datos referentes al COVID-19, seguido de las redes sociales. A su vez, se menciona que, en un estudio realizado por el Instituto para el Desarrollo Rural de Sudamérica en zonas rurales y urbanas de Bolivia, concluyó que, en zonas rurales, se vio un gran número de personas que acceden a la información a través las emisoras radiales. Contrario a las zonas urbanas, en donde la televisión es el medio más

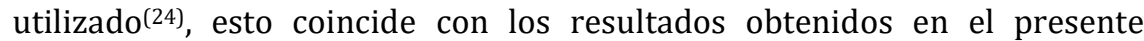
trabajo.

Es así que, con respecto a las informaciones sobre el COVID-19, el 100\% de los encuestados en las tres comunidades ha manifestado que sí tiene conocimientos básicos y recibe información sobre el Coronavirus. Sobre este punto es importante resaltar que, en todas las comunidades los encuestados han coincidido que las informaciones y noticias sobre el COVID-19 las recibe principalmente a través de la radio. De igual modo, el segundo medio por el cual se informan es la televisión, seguida de las redes sociales (Figuras 12 y 13).

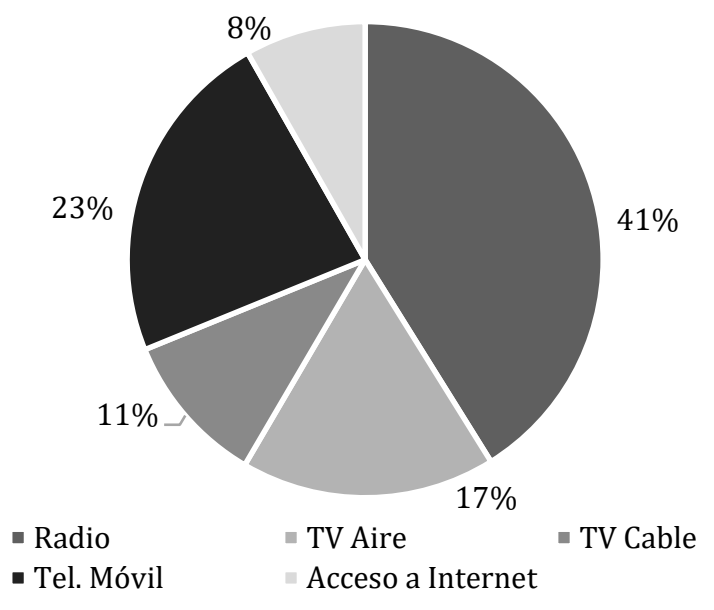

Figura 12. Medios de Comunicación más Utilizados en las 3 comunidades, 2021. 


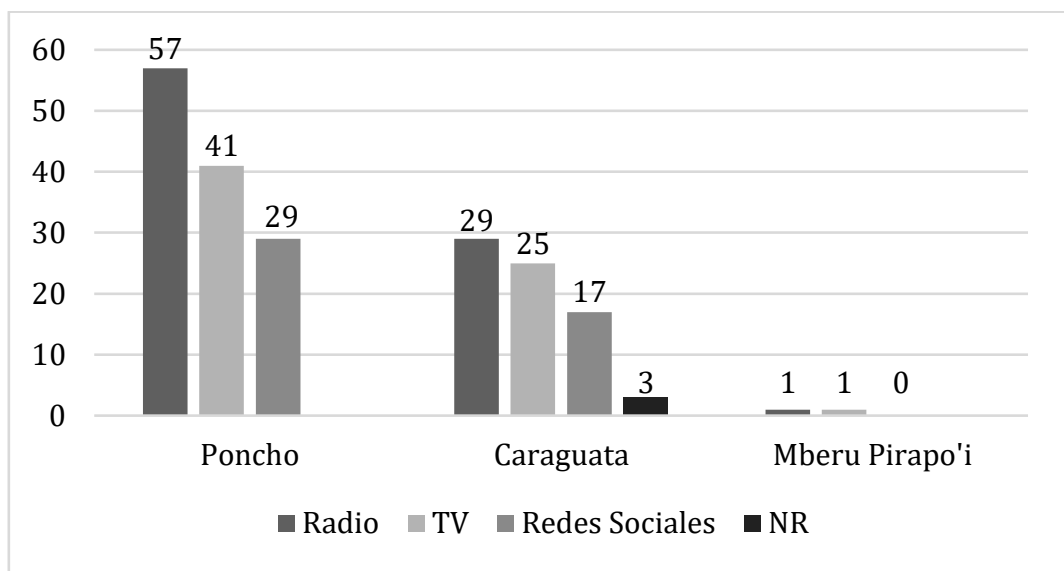

Figura 13. Medios de Comunicación por los que se Recibe Información sobre el COVID-19, en las 3 comunidades, 2021.

En relación a la forma en la que se realiza la divulgación de la información sobre el COVID-19, gran parte de los entrevistados (64\%) indicó que sí existen informaciones claras y seguras; mientras que el 22\%, manifestó que esta no fue suficiente ni clara. Sin embargo, el restante $14 \%$ de los entrevistados se informaban a través de las redes sociales, y comentaron que la información disponible era confusa y no siempre confiables, estos resultados coinciden con los resultados de la encuesta de percepciones sobre el COVID-19 en Paraguay(23).

De igual modo, se menciona que, en su mayoría estos referentes no conocen otros actores que no sean el personal del Puesto de salud como agentes diseminadores de la información, un número menor identifican a los líderes locales como posibles diseminadores de la información. Se resalta que, los representantes de centros religiosos fueron importantes difusores de la información. En el caso de las comunidades indígenas estas tuvieron charlas por parte de la Secretaría de Asuntos Indígenas de la Gobernación de Itapúa.

Así también, los representantes de los medios de comunicación de la zona, concordaron que las radios y demás tipo de medios que sean locales son bastante influyentes en la comunidad, principalmente la radio. Y en relación a capacitaciones o talleres sobre la comunicación y difusión en tiempos de pandemia, han respondido que no han accedido a nada concreto, pero que 
han sido partícipes de reuniones ofrecidas en el local del Puesto de Salud de Alto Verá.

Los comunicadores concuerdan que el objetivo primordial de sus medios fue informar constantemente sobre los protocolos sanitarios que debían ser implementados (distanciamiento social, cuarentena, lavado de manos, uso de alcohol en gel, etc.) y que, en el caso radial, han optado por la grabación de segmentos recordatorios que compartían durante los inicios de la pandemia (meses de marzo a julio), pero también así indican que la socialización de dichos segmentos ha disminuido.

Los líderes y referentes claves indican que no identificaron en el proceso programas de capacitación para la comunidad en cómo prevenir la pandemia por parte de organizaciones gubernamentales ni de otra índole. Los medios de comunicación radiales fueron utilizados en todo el distrito, esto espacios fueron sumamente útiles, siendo considerados herramientas fundamentales para para que la población accediera a la información relacionada a la pandemia para evitar contagios, se destaca los espacios proveídos por estos para que el personal de blanco pueda informar sobre la situación de la Pandemia (COVID-19).

\section{CONCLUSIÓN}

Las entrevistas a actores claves de la comunidad indicaron que no identificaron en el proceso programas de capacitación para la comunidad sobre cómo prevenir el contagio del COVID-19 por parte de organizaciones gubernamentales ni de otra índole. Los principales actores para la difusión de la información en el área fueron los medios radiales, y en parte, por los líderes comunitarios, a partir de la información proveída por personal del Puesto de Salud/MSyBS.

Se evidencia escasa conciencia sobre el peligro que conlleva la pandemia del COVID-19, a pesar de contar mínimamente con los servicios básicos, se pudo identificar que la población ha dejado de lado las medidas sanitarias establecidas por el MSyBS y la situación de relajo puede deberse a que a) el área registró escasos casos de personas infectadas en con el coronavirus, b) la falta de información o falta de conciencia del poder de infestación del virus y su rápida dispersión una vez que ingrese a un área.

En la comunidad indígena de Mberú Pirapo'i, se pudo identificar escasa aplicación de protocolos sanitarios, el uso de tapabocas es la única medida de prevención que utilizan para prevenir el virus. Se identificó que no existe una relación directa entre la comunidad indígena y las comunidades a su 
alrededor, estos tienen un vínculo más directo con autoridades departamentales. Se resalta que el Puesto de Salud/MSyBS ha desarrollado actividades de concienciación sobre el COVID-19 en las comunidades indígenas

Se destaca que los medios radiales son el instrumento más importante para diseminar la información a las comunidades, sin embargo, la manera en la que la información es difundida no es la adecuada. Los representantes de medios admiten en que es posible acordar oportunidades de capacitación en orden a mejorar el estilo, enfoque y rasgos generales del discurso comunicativo frente a la pandemia en la comunidad y, en particular, para lograr que la misma sea masiva, flexible y accesible para las comunidades rurales.

Finalmente, se puede concluir que, las características culturales de las zonas rurales y en especial, de las comunidades indígenas son factores esenciales que se deben tener en cuenta para que la comunicación de la información sobre el COVID-19 sea efectiva y adquirida por estas comunidades más vulnerables.

\section{AGRADECIMIENTO}

Este proyecto es financiado por el Consejo Nacional de Ciencia y Tecnología del Paraguay (CONACYT) a través del Programa PROCIENCIA con recursos del Fondo para la Excelencia e Investigación - FEEI.

\section{PARTICIPACIÓN DE AUTORES}

AE: conceptualización de las ideas, así como formulación de los objetivos de la investigación. Además, dirección general del proyecto e investigadora principal, diseño y desarrollo de la metodología de la investigación. Aportes significativos en la validación de los datos y redacción del borrador final, así como en la revisión y edición; BF: preparación de la visualización de los datos, redacción del borrador final; revisión y edición del documento; LW: recopilación y organización de la información.

\section{CONFLICTO DE INTERESES}

Los autores manifiestan no poseer conflictos de interés respecto de su contenido. 


\section{REFERENCIAS BIBLIOGRÁFICAS}

1. OMS. Orientaciones para el público [Online]; 2019. Available in: https://bit.ly/38yvKX8

2. OMS. Nuevo Coronavirus 2019 [Online]; 2019. Available in: https://bit.ly/2N9Vxxi

3. GOOGLE. Alerta sobre el Covid-19 [Online]; 2020. Available in: https://bit.ly/38zYAX5

4. BBC NEWS|MUNDO. BBC News Mundo [Online]; 2019. Available in: https://www.bbc.com/mundo/noticias-america-latina-53085660.

5. Moreno 0. La Divulgación de la información en la encrucijada de la crisis del COVID-19 en Paraguay. Sociedad Científica del Paraguay. 2020; 25(1):58-85.

6. Díaz L, Torruco U, Martínez M. La entrevista, recurso flexible y dinámico. ELSEVIER. 2013 Septiembre;2:162-167.

7. Guber R. La etnografía:métodos, campo y reflexividad Bogotá: Siglo Veintiuno; 2001.

8. Spicker P, Alvarez S, Gordon D. Glosario.

9. FAO en Paraguay. Paraguay en una mirada [Online]; 2021. Available in: https://bit.ly/20h50DN

10.Villatoro P. Indicadores no Monetarios de Privación en América Latina: Disponibilidad, Comparabilidad y Pertinencia. Santiago: Comisión Económica para América Latina y el Caribe; 2017. Available in: https://bit.ly/3a2JxVS

11.CEPAL/UNICEF. Pobreza Infantil en América Latina y el Caribe.; 2010. Available in: https://bit.ly/3qPAVZo

12.STP. Plan Nacional de Desarrollo 2030.; 2014. Available in: https://bit.ly/3taQ1Ke

13.OMS/UNICEF. Agua, saneamiento, higiene y gestión de desechos en relación con el SARS-CoV-2, el virus causante de la COVID-19; 2020. Available in: https://bit.ly/2YM0nTA

14.ONU. Objetivos de Desarrollo Sostenible [Online]. Available in: https://bit.ly/3c6sBPc

15. Paraguay Sostenible. Agua limpia y saneamiento [Online]. Available in: https://bit.ly/3qduHSw

16. Ministerio de Salud Pública y Bienestar Social. COVID-19: ¿Quiénes son la población de riesgo y qué deben hacer? [Online].; 2020. Available in: https://bit.ly/3prOGNy

17. Ministerio de Salud Pública y Bienestar Social. COVID-19: lavado de manos y desinfección de superficies como medidas de prevención [Online]; 2020. Available in: https://bit.ly/3oxAce4 
18. Ministerio de Salud Pública y Bienestar Social. Antecedentes regulatoios [Online].; 2020. Available in: https://bit.ly/20NwnoQ

19. Ministerio de Salud Pública y Bienestar Social. Resoluciones COVID-19 [Online].; 2020. Available in: https://www.mspbs.gov.py/resolucionescovid19.html

20.DESA - ONU. Pueblos Indígenas y la Pandemia del COVID-19. Departamento de Asuntos Económicos y Sociales de las Naciones Unidas; 2020. Available in: https://bit.ly/20qXCVM

21. Ley $\mathrm{N}^{\circ}$ 5469/2015, De la salud de los pueblos indígenas [Online]; 2015. Available in: https://bit.ly/30BoXrh

22. DINASAPI. Guía y Recomendaciones para la Prevención y Protección del COVID-19 Dirigidos a los Pueblos y Comunidades Indígenas del Paraguay; 2020. Available in: https://bit.ly/3kUM5dF

23. Ministerio de Tecnologías de la Información y Comunicación. La TV es el principal medio de consumo de la información sobre COVID-19 en Paraguay, revela encuesta [Online]; 2020. Available in: https://bit.ly/3qur2jc

24.IPDRS. ¿Cómo recibe la información sobre el Covid-19 la población rural y urbana en Bolivia?; 2020. Available in: https://bit.ly/3v84ZT7

25. Hernádez Sampieri R, Fernández C, Baptista P. Metodología de la Investigación. México: McGrall - Hill/Interamericana Editores; 2014. 